\title{
Pilot-Based Carrier Frequency Offset Estimation in OFDM Systems
}

\author{
Cheng Li and Tung-Sang Ng, Fellow, IEEE \\ Dept. of Electrical and Electronic Engineering, The University of Hong Kong \\ Email: \{chengli,tsng\}@eee.hku.hk
}

\begin{abstract}
The ability of OFDM to mitigate channel frequency selectivity is impaired by its vulnerability to carrier frequency offset (CFO). In this paper, a new pilot-based carrier frequency offset estimation scheme for frequency selective slowly-varying channels is proposed. This scheme exploits the inherent redundancy introduced by the OFDM signaling and no additional training symbol is needed. The synchronization process is to minimize a LMS-like cost function, which essentially quantifies the variance of the pilot tones across several OFDM symbols. Moreover, a reduced-complexity estimator is also developed to reduce the implementation complexity. The CFO estimation range can span the whole OFDM signal bandwidth by non-uniformly distributing the embedded pilot tones in the frequency domain. Computer simulation results show that the performance of the proposed estimator can meet the requirement of typical OFDM communication systems.
\end{abstract}

\section{Introduction}

Since it was first proposed in 1966, orthogonal frequency division multiplexing (OFDM) has received extensive interest in wireless broadband communications for its good performance in frequency selective channels. However this advantage is impaired by its high sensitivity to carrier frequency offset, which destroys the mutual orthogonality between subcarriers and degrades the system performance severely [1]. Consequently, accurate estimation and correction of CFO is required at the receiver before OFDM demodulation.

Works on the CFO estimation for OFDM systems can be roughly divided into two categories: data aided and nondata aided (blind or semi-blind) schemes. Data aided schemes employ training sequences that are known to the receiver [2], while non-data aided schemes exploit the redundant information contained in the received data sequences, such as cyclic prefix (CP) [3] and virtual carrier (VC) [4] to estimate the CFO. Recent works appear to focus on non-data aided CFO estimation methods due to its transmission efficiency.

In most practical OFDM communication systems, such as IEEE 802.11a WLAN, known pilot tones are spread out in the time-frequency grid and are primarily used for channel estimation. In this paper, we take advantage of these pilot tones and propose a LMS-like cost function. By minimizing this cost function the CFO estimate is obtained. In this scheme no additional training symbol is needed and consequently the transmission efficiency is not affected. The rest of this paper is organized as follows. In section 2 , we develop a matrix-vector model for the discrete-time baseband equivalent OFDM system. The pilot-based CFO estimation algorithm is described in section 3 and the reduced-complexity estimator is also presented in this section. Computer simulation results are given in section 4 and Section 5 concludes this paper.

\section{System Model}

Let $\mathbf{S}_{i} \triangleq\left[\begin{array}{llll}S_{i}(1) & S_{i}(2) & \cdots & S_{i}(M)\end{array}\right]^{T}$ denote the $i$ th block of data to be transmitted and the data is assumed to be independently identically distributed (i.i.d.) random variables with zero mean and convariance matrix

$$
R_{S S}(i, j)=E\left\{\mathbf{S}_{i} \mathbf{S}_{j}^{H}\right\}=\left\{\begin{array}{c}
\mathbf{0}_{M}, i \neq j \\
\sigma_{S}^{2} \mathbf{I}_{M}, i=j
\end{array},\right.
$$

where $(\cdot)^{T}$ and $(\cdot)^{H}$ denote transpose and complex conjugate transpose respectively, $\mathbf{0}_{M}$ and $\mathbf{I}_{M}$ are $M \times M$ zero and identity matrices.

Let $\mathbf{P}_{i} \triangleq\left[\begin{array}{llll}P_{i}(1) & P_{i}(2) & \cdots & P_{i}\left(N_{P}\right)\end{array}\right]^{T}$ represent the pilot vector that is attached to the $i$ th data block, where $P_{i}(m)$ is the $m$ th pilot tone in the $i$ th block and $N_{P}$ is the number of pilot tones in one OFDM symbol. We denote the indexes of data bearing and pilot subcarriers by the sets of $V=\left\{v_{k}\right\}_{k=1}^{M}$ and $U=\left\{u_{k}\right\}_{k=1}^{N_{p}}$, respectively. After pilot insertion the data block to be transmitted can be written as:

$$
\mathbf{X}_{i} \triangleq\left[\begin{array}{llll}
X_{i}(1) & X_{i}(2) & \cdots & X_{i}(N)
\end{array}\right]^{T},
$$

where we have $X_{i}\left(v_{k}\right)=S_{i}(k)$ and $X_{i}\left(u_{k}\right)=P_{i}(k)$.

An $N$-point inverse DFT (IDFT) is then applied to $\mathbf{X}_{i}$ in (2), resulting in the matrix representation

$\mathbf{x}_{i}=\mathbf{F}_{i}^{H} \mathbf{X}_{i}$,
where $\left[\mathbf{F}_{N}\right]_{m, n}=N^{-(1 / 2)} \exp (-j 2 \pi m n / N)$ is the DFT matrix.

After IDFT, the last $L_{1}$ samples of the OFDM symbol are duplicated and inserted at the beginning of the OFDM symbol to form the CP. Since the CP is discarded at the 
receiver and not used in our algorithm, we do not model it in the formulation.

Let $\varepsilon_{0}$ denote the normalized CFO, which is the actual CFO divided by the subcarrier spacing $\Delta f$. After removing the $\mathrm{CP}$, the received ith OFDM symbol can be represented by

$$
\mathbf{r}_{i}=e^{j 2 \pi \varepsilon_{0}\left(L_{i}+i\left(N+L_{i}\right)\right) / N} \Omega\left(\varepsilon_{0}\right) \mathbf{F}_{N}^{H} \mathbf{H} \mathbf{X}_{i}+\mathbf{w}_{i}
$$

where $\Omega\left(\varepsilon_{0}\right)=\operatorname{diag}\left(\begin{array}{llll}1 & e^{j 2 \pi \varepsilon_{0} / N} & \cdots & e^{j 2 \pi \varepsilon_{0}(N-1) / N}\end{array}\right) \quad$ and $\mathbf{w}_{i} \triangleq\left[\begin{array}{llll}w_{i}(1) & w_{i}(2) & \cdots & w_{i}(N)\end{array}\right]^{T}$ is white Gaussian noise vector with power $\sigma_{w}^{2}$. In (4), $\mathbf{H}=\operatorname{diag}(H(1), H(2), \cdots, H(N))$ is a diagonal matrix with diagonal elements containing the channel frequency response on the corresponding subcarriers. We assume the channel is time invariant during one OFDM packet that contains $K$ consecutive OFDM symbols.

\section{A Pilot-Based Carrier Frequency Offset Estimation Algorithm}

\subsection{Derivation of the Estimator}

At the receiver, before the demodulation by DFT, the CFO is compensated by the trial value of $\varepsilon_{0}$, which is denoted by $\tilde{\varepsilon}$. It follows from (4) that the CFO compensated OFDM symbol becomes

$$
\tilde{\mathbf{r}}_{i}=e^{-j 2 \pi \tilde{\varepsilon}\left(L_{1}+i\left(N+L_{1}\right)\right) / N} \Omega^{H}(\tilde{\varepsilon}) \mathbf{r}_{i}
$$

and the compensated received data symbol after DFT is:

$$
\tilde{\mathbf{R}}_{i} \triangleq\left[\begin{array}{llll}
\tilde{R}_{i}(1 ; \tilde{\varepsilon}) & \tilde{R}_{i}(2 ; \tilde{\varepsilon}) & \cdots & \tilde{R}_{i}(N ; \tilde{\varepsilon})
\end{array}\right]^{\mathrm{T}}=\mathbf{F}_{N} \tilde{\mathbf{r}}_{i} .
$$

Denote $\Delta \varepsilon=\varepsilon_{0}-\tilde{\varepsilon}$ as the residual CFO after CFO compensation. The $k$ th entry of $\tilde{\mathbf{R}}_{i}$ can be rewritten as:

$$
\begin{aligned}
\tilde{R}_{i}(k ; \tilde{\varepsilon}) & =\mathbf{f}_{u_{k}} \tilde{\mathbf{r}}_{i} \\
& =e^{\frac{j 2 \pi \tilde{z}\left(4+i\left(N+L_{1}\right)\right)}{N}} \frac{1}{\sqrt{N}} \sum_{n=0}^{N-1} r_{i}(n) e^{-\frac{j 2 \pi n(k+\tilde{\varepsilon})}{N},}
\end{aligned}
$$

with $\mathbf{f}_{k}$ being the kth row of $\mathbf{F}_{N}$. After some straightforward manipulations, (7) can be rewritten as:

$$
\tilde{R}_{i}(k ; \tilde{\varepsilon})=e^{\frac{j 2 \pi \Delta \varepsilon\left(L_{1}+i\left(N+L_{1}\right)\right)}{N}}\left[\sum_{m=0}^{N-1} I_{m-k}(\Delta \varepsilon) H(m) X_{i}(m)\right]+\tilde{W}_{i}(k)
$$

where

$$
I_{m}(\varepsilon)=\frac{1}{N} \sum_{n=0}^{N-1} e^{j 2 \pi n(m+\varepsilon) / N} .
$$

and $\tilde{W}_{i}(k)$ is the $k$ th entry of the noise vector after DFT.

It follows that the variance of $\tilde{R}_{l}(k ; \tilde{\varepsilon})$ on the $k$ th pilot subcarrier is:
$\operatorname{Var}\left\{\tilde{R}_{i}\left(u_{k} ; \tilde{\varepsilon}\right)\right\}=\sigma_{s}^{2} \sum_{m=0, m \in U}^{N-1}|H(m)|^{2}\left|I_{m-u_{k}}(\Delta \varepsilon)\right|^{2}+\sigma_{w}^{2}, u_{k} \in U$

The variance of $\tilde{R}_{i}\left(u_{k} ; \tilde{\varepsilon}\right)$ reaches its minimum when $\Delta \varepsilon=0$, i.e., $\tilde{\varepsilon}=\varepsilon_{0}$. It follows that (10) can be used as a criterion for minimization to obtain $\varepsilon_{0}$. In practical implementation, the variance is replaced by its natural estimator: the sample variance. The sample variances across $K$ consecutive symbols on $N_{P}$ pilot subcarriers are computed and averaged to obtain the following cost function:

$C_{1}(\tilde{\varepsilon})=\frac{1}{N_{P} K} \sum_{k=1}^{N_{P}}\left\{\sum_{i=0}^{K-1}\left|\frac{\tilde{R}_{i}\left(u_{k} ; \tilde{\varepsilon}\right)}{P_{i}(k)}\right|^{2}-\left|\frac{1}{K} \sum_{i=0}^{K-1} \frac{\tilde{R}_{i}\left(u_{k} ; \tilde{\varepsilon}\right)}{P_{i}(k)}\right|^{2}\right\}$.

To alleviate the effects of frequency selectivity, we revise the cost function (11) to be

$$
C(\tilde{\varepsilon})=\frac{1}{N_{P}} \sum_{k=1}^{N_{P}} \frac{\sum_{i=0}^{K-1}\left|\frac{\tilde{R}_{i}\left(u_{k} ; \tilde{\varepsilon}\right)}{P_{i}(k)}\right|^{2}-\left|\frac{1}{K} \sum_{i=0}^{K-1} \frac{\tilde{R}_{i}\left(u_{k} ; \tilde{\varepsilon}\right)}{P_{i}(k)}\right|^{2}}{\sum_{i=0}^{K-1}\left|\frac{\tilde{R}_{i}\left(u_{k} ; \tilde{\varepsilon}\right)}{P_{i}(k)}\right|^{2}} .
$$

The revised cost function essentially measures the normalized variance by dividing the former cost function by the power on the $k$ th pilot subcarrier.

Our objective is to find $\tilde{\varepsilon}$ that minimizes (12). Therefore the estimator is:

$$
\hat{\varepsilon}=\arg \min _{\tilde{\varepsilon}} C(\tilde{\varepsilon}) .
$$

If the $\mathrm{VC}$ is regarded as the pilot that transmits zeros, (12) becomes a general extension of the cost function (4) in [4]. In this case, however, some modifications to (12) are need because $P_{i}(k)=0$. In this particular case, the variance is actually the average power of the pilot tones because the ICI on the pilot subcarrier is approximated as Gaussian noise with zero mean. As a result, the cost function takes the following form,

$$
\begin{aligned}
C_{2}(\tilde{\varepsilon}) & =\frac{1}{N_{P} K} \sum_{k=1}^{N_{P}} \sum_{i=0}^{K-1}\left|\tilde{R}_{i}\left(u_{k} ; \tilde{\varepsilon}\right)\right|^{2} \\
& =\frac{1}{N_{P} K} \sum_{k=1}^{N_{P}} \sum_{i=0}^{K-1} \mathbf{f}_{u_{k}} \tilde{\mathbf{r}}_{i} \tilde{\mathbf{r}}_{i}^{H} \mathbf{f}_{u_{k}}^{H} \\
& =\frac{1}{N_{P} K} \sum_{k=1}^{N_{P}} \sum_{i=0}^{K-1} \mathbf{f}_{u_{k}} \Omega^{H}(\tilde{\varepsilon}) \mathbf{r}_{i} \mathbf{r}_{i}^{H} \Omega(\tilde{\varepsilon}) \mathbf{f}_{u_{k}}^{H}
\end{aligned}
$$

It is found that the above cost function is exactly in the same form as (4) in [4] except for different notations. The performance of the proposed algorithm in real-life implementation outperforms that of [4] because both the pilot tones and virtual carriers can be exploited in the proposed algorithm. Of course, this benefit is obtained at the cost of increased complexity.

\subsection{Estimation Range and Pilot-Tone Placement}


To investigate the effect of the pilot-tone arrangement on the estimation range, we consider a particular case first. In this case, the pilot tones are uniformly distributed over all the subcarriers. The CFO on the received signal shifts the subcarriers along with introducing ICI as illustrated by (7) and (8). It follows that in the absence of noise $\tilde{R}_{l}\left(u_{k} ; \tilde{\varepsilon}\right)$ and $C(\tilde{\varepsilon})$ is periodic with period $N / N_{P}$ and hence the estimation range of the estimator is $\left[-N \Delta f / 2 N_{P}, N \Delta f / 2 N_{P}\right] \mathrm{Hz}$, which is a multiple of subcarrier spacing.

In order to extend the estimation range to the whole OFDM signal bandwidth, it is required to non-uniformly arrange the pilot tones to make (12) reach its maximum period N. However, if $\operatorname{Var}\left\{\tilde{R}_{l}\left(u_{k} ; \varepsilon_{0}\right)\right\}$ reaches the minimum, (10) indicates that $\operatorname{Var}\left\{\tilde{R}_{i}\left(u_{k} ; \varepsilon_{0}+\Delta u\right)\right\}$ also reaches the minimum value, where $\Delta u$ is the spacing between the $k$ th pilot subcarrier and any other pilot subcarrier. Although this ambiguity does not affect the estimation range due to averaging over all the subcarrier in the cost function, the estimator performance is affected because some local minima appear in the cost function. In order to suppress this ambiguity, we need to arrange the pilot tones so that the spacing between any two the pilot subcarrier are not equal, i.e.,

$\bmod \left[\left(u_{k}-u_{l}\right), N\right] \neq \bmod \left[\left(u_{k^{\prime}}-u_{l^{\prime}}\right), N\right]$, for $\{k, l\} \neq\left\{k^{\prime}, l^{\prime}\right\}$

where $\bmod (x, y)$ is the remainder after dividing $x$ by $y$.

\subsection{Derivation of the Reduced-Complexity Estimator}

The cost function (12) is computation demanding and the main computation load is on computing $\tilde{R}_{i}(k ; \tilde{\varepsilon})$. From (7) we see that an $N$-point FFT must be performed to obtain $\tilde{R}_{i}(k ; \tilde{\varepsilon})$. It follows that a total of $N_{p} K N$-point FFT operations are needed to compute the cost function at every trial value $\tilde{\varepsilon}$ and the computational complexity also depends on the step-size. In the following we shall derive an algorithm analogous to that proposed in [5]. This algorithm provides a good tradeoff between estimation accuracy and implementation complexity.

It is apparent that (7) consists of a Fourier transform for the discrete-time signal $r_{i}(n)$ and a phase rotation. It is known that the Fourier transform for discrete-time signal can be approximated by the sampled version of the DFT. In order to improve the accuracy, zeros are padded to the tail of $r_{i}(n)$ to form a zero-padded sequence $\left\{r_{i}^{\prime}(n)\right\}$ :

$$
r_{i}^{\prime}(n)=\left\{\begin{array}{ll}
r_{i}(n), & 0 \leq n \leq N-1 \\
0, & N \leq n \leq Q N-1
\end{array},\right.
$$

where $Q$ is called the oversize ratio and usually selected to be an integer power of 2 .
Next a $Q N$-point FFT is applied to $\left\{r_{i}^{\prime}(n)\right\}$ to form

$$
\mathbb{R}_{i}(\omega)=\sum_{n=0}^{Q N-1} r_{i}^{\prime}(n) e^{\frac{j 2 \pi n \omega}{Q^{N}}}, \omega \in Z,
$$

where $\mathrm{Z}$ denotes the set of integers.

$$
\text { It follows that } \tilde{R}_{f}\left(u_{k} ; \tilde{\varepsilon}\right) \text { can be approximated by }
$$

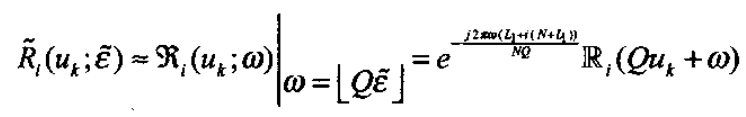

where $[\cdot]$ is the operator that rounds a real number to its nearest integer. Substituting (18) into (12) leads to the reduced-complexity estimator:

$$
\hat{\varepsilon} \approx \arg \min _{\omega} \frac{1}{Q N_{P}} \sum_{k=1}^{N_{p}} \frac{\sum_{i=0}^{K-1}\left|\frac{\Re_{i}\left(u_{k} ; \omega\right)}{P_{i}(k)}\right|^{2}-\left|\frac{1}{K} \sum_{i=0}^{K-1} \frac{\Re_{i}\left(u_{k} ; \omega\right)}{P_{i}(k)}\right|^{2}}{\sum_{i=0}^{K-1}\left|\frac{\Re_{i}\left(u_{k} ; \omega\right)}{P_{i}(k)}\right|^{2}}
$$

Comparing (12) with (19), it is obvious that the former continuous $\hat{\varepsilon}$ is approximated by discrete points $\omega / Q$ and only $N_{P} K \quad Q N$-point $\mathrm{FFT}$ operations are needed to compute the cost function for all trial values. The reducedcomplexity estimator introduces a discretization error that is dependent on the over size ratio $Q$ [5]. By selecting larger $Q$ and $\mathrm{K}$ in (19), better performance can be achieved at the cost of increased computational complexity and vice versa.

\section{Simulation Results}

In this section, computer simulation results as a function of various system parameters are given for investigating the estimator performance. The following parameters are employed in the simulation: the number of subcarriers $N=32$, the length of CP $L_{1}=16$, the CFO $\varepsilon_{0}=0.2571$, the packet length $K=10$. Four pilot tones transmitting values of one are employed in every OFDM symbol. The pilot tones are non-uniformly distributed in the subcarriers and their positions are $\{1,8,17,28\}$. The information bits are generated randomly and mapped onto 4 psk modulation constellation. The signal to noise ratio (SNR) is defined as $S N R=E\left(\left|S_{i}(k)\right|^{2}\right) / \sigma_{w}^{2}=\sigma_{s}^{2} / \sigma_{w}^{2} \quad$. Uncorrelated scattering Rayleigh fading channel with exponential decaying power delay profile is considered in the simulation. The maximum channel delay spread is smaller than the length of CP. The channel is assumed to be static within one OFDM packet and is generated independently for each packet.

Fig. 1 plots the mean square error (MSE) of the CFO estimate obtained by running 3000 realizations, together with the modified Cramér-Rao bound (MCRB) [6] as a benchmark. It is observed that the MSE reaches an error 
floor when SNR is greater than 10dB. This is due to the limit resolution of the frequency-domain sampling by DFT and the error floor can be lowered by increasing the oversize ratio $Q$. Also depicted in Fig. 1 is the MSE performance obtained through the CP-based estimator in [3]. It is apparent that the proposed reduced-complexity estimator provides a performance comparable to that of the CP-based estimator of [3] at median-to-high SNR even with $Q=4$ and the proposed estimator is superior to the $\mathrm{CP}$ based estimator with $Q=128$. In addition, the performance of the CP-based estimator depends on the length of CP and degrades when the delay spread of the dispersive channel increases. Similar error floor phenomenon also occurs in the case of the CP-based estimator.

Another advantage over the CP-based estimator is that the estimation range of the proposed estimator can span the entire OFDM signal bandwidth, which is shown by the results in Table I. On the other hand, the estimation range of the CP-based estimator of [3] is limited to the subcarrier spacing. It is also observed that when the true CFO is an integer, there is no discretizaiton error. In the case of non integer true CFO, the discretization error do exist and it can be reduced by increasing the over size ratio $Q$.

Fig. 2 shows that the MSE performance of the proposed estimator can be improved by increasing the packet length $K$ at the cost of increased complexity. The turning point at which the error floor occurs is lowered by selecting a larger $K$. Simulation conditions are the same as that of Fig. 1.

\section{Discussion and Conclusion}

This paper has proposed a novel pilot-based CFO estimation scheme for OFDM over frequency selective slowly-varying channels. The underlying rationale of the proposed algorithm is based on the fact that the known pilot tones are deterministc along the time axis in the absence of CFO and the variance of the pilot tones should be the noise power. However, the presence of the CFO introduces ICI on the pilot subcarrier and hence increases the variance of the pilot tones. Consequently, the CFO estimation is attained by minimizing a LMS-like cost function, which essentially computes the variance of the pilot tones across several OFDM symbols. The cost function is a general extension to that in [4]. A reducedcomplexity estimator employing the zero-padding FFT technique has also been developed for practical implementation of the proposed algorithm. Computer simulation results show that the proposed estimator can yield good performance in median to high SNR range. In addition, it is shown that the proposed algorithm outperforms the CP-based algorithm of [3] in terms of MSE of the CFO estimate.

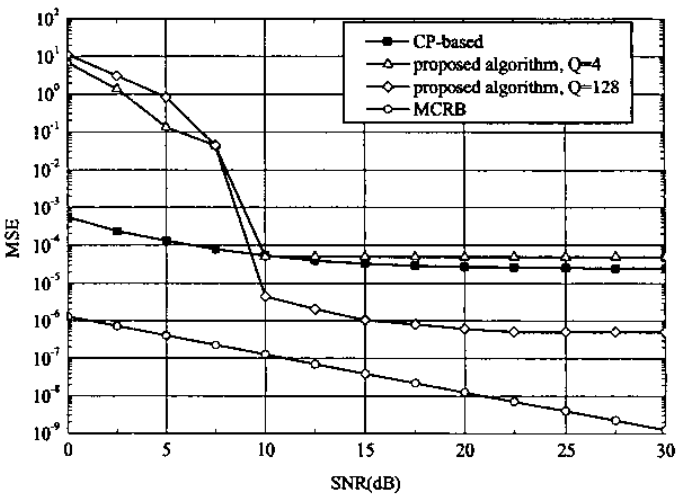

Figure 1. Mean Square Error against SNR with $K=10$

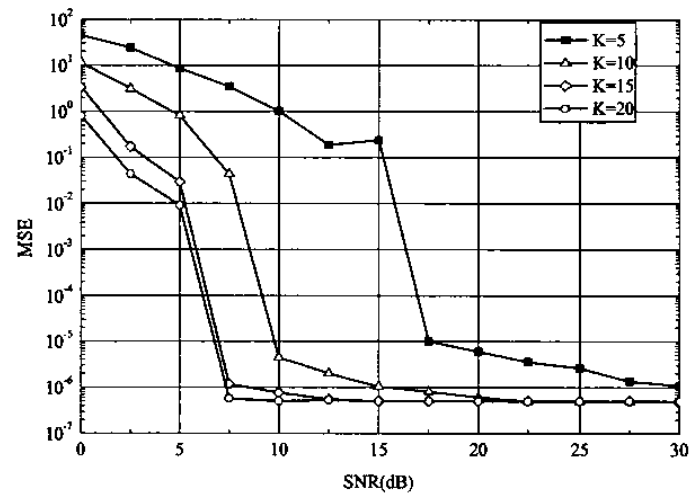

Figure 2. Mean Square Error against SNR by varying the OFDM packet length $K$ with $Q=128$

Table I. The CFO estimate when $K=10$ and SNR=20dB

\begin{tabular}{|c|c|c|c|}
\hline$\varepsilon_{0} Q$ & 16 & 64 & 128 \\
\hline-15.83 & -15.8125 & -15.8281 & -15.8281 \\
\hline-11.213 & -11.1875 & -11.2188 & -11.2109 \\
\hline-7.717 & -7.6875 & -7.7188 & -7.7188 \\
\hline-3 & -3.0000 & -3.0000 & -3.0000 \\
\hline 1.1791 & 1.1875 & 1.1875 & 1.1797 \\
\hline 5.2571 & 5.2500 & 5.2500 & 5.2578 \\
\hline 9.337 & 9.3125 & 9.3438 & 9.3359 \\
\hline 16 & 16.0000 & 16.0000 & 16.0000 \\
\hline
\end{tabular}




\section{References}

[1] T. Pollet, M. Van Bladel, and M. Moenaclaey, "BER sensitivity of OFDM systems to carrier frequency offset and Wiener phase noise," IEEE Trans. Commun., vol. 43, pp. 191-192, Feb./Mar./Apr. 1995.

[2] T. M. Schmidl and D. C. Cox, "Robust frequency and timing synchronization for OFDM," IEEE Trans. Commun., vol. 45, no. 12, pp. 1613-1621, Dec. 1997.

[3] J.-J. van de Beek, M. Sandell, and P.-O. Börjesson, "ML estimation of timing and frequency offset in OFDM systems," IEEE Trans. Signal Processing, vol. 45, pp. 1800-1805, July 1997.

[4] H. Liu and U. Tureli, "A high efficiency carrier estimator for OFDM communications," IEEE Commun. Lett., vol. 2, pp. 104-106, Apr. 1998.

[5] Jing Lei and Tung-Sang $\mathrm{Ng}$, “A robust OFDM carrier offset estimator based on distinctively-spaced pilottones." to appear in IEEE Trans. Wireless Commun.

[6] The modified Cramer-Rao bound and its application to synchronization problems D'Andrea, A.N.; Mengali, U.; Reggiannini, R. Communications, IEEE Transactions on , February-April 1994 Page(s): 1391 1399 